\title{
Use of HINTS in the acute vestibular syndrome. An Overview
}

Jorge C Kattah

To cite: Kattah JC. Use of HINTS in the acute vestibular syndrome. An Overview. Stroke and Vascular Neurology 2018;3: e000160. doi:10.1136/svn2018-000160

Received 5 April 2018 Revised 18 May 2018 Accepted 24 May 2018 Published Online First 23 June 2018

Check for updates

Department of Neurology, University of Illinois College of Medicine, Peoria, Illinois, USA

Correspondence to Dr Jorge C Kattah; kattahj@uic.edu

\section{ABSTRACT}

Following the initial description of HINTS to diagnose acute vestibular syndrome (AVS) in 2009, there has been significant interest in the systematic evaluation of HINTS to diagnose stroke and other less common central causes of AVS. This trend increased with availability of the video head impulse test (video-HIT). This article reviews the original papers and discusses the main publications from 2009 to 2017. Many authors use video-HIT in the diagnosis of patients with AVS; this paper focuses on the major publications on the topic featuring nystagmus, manual and video-HIT, and skew deviation. Twenty-five papers provide a summary of the last 8 years' application of HINTS, the video-HIT added quantitative information to the early clinical observations. Further research will undoubtedly provide specific combination of abnormalities with high degree of lesion localisation and aetiology. In a short time following the original description, neurotologist and neurologists in the evaluation of AVS use the HINTS triad. The introduction of the video-HIT added greater understanding of the complex interaction between the primary vestibular afferents, brainstem and cerebellum. In addition, it permits evaluation of the angular vestibuloocular reflex in the plane of all six semicircular canals, with accurate peripheral versus central lesion localisation often corroborated by brain imaging.

\section{DEFINITION AND EPIDEMIOLOGY}

The acute vestibular syndrome (AVS) is defined as the sudden onset of acute, 'continuous' vertigo (lasting longer than 24 hours), associated with nausea, vomiting and head motion intolerance. The term was initially introduced by Hotson and Baloh in 1998. ${ }^{1}$ Vestibular neuritis (VN), the most common cause of an AVS, has an estimated annual incidence of 3.5 per 100000 populations. It is also estimated that $10 \%-20 \%$ of dizzy patients visiting emergency departments (ED) have an AVS which corresponds to 25000 to 500000 annual ED visits in the USA alone. ${ }^{2}$ The second most common cause of an AVS is an ischaemic stroke involving the cerebellum or the brainstem (probably $5 \%-10 \%$ of all patients with AVS). ${ }^{3}$ Vertigo and nystagmus ranked as the most common symptom/sign in patients with posterior circulation stroke in The New England Posterior Circulation Registry. ${ }^{45}$ Pertinent to the subject of this chapter, I will focus on the AVS with VN as the most common cause of continuous vertigo and will also focus on vascular vertigo, which a common VN mimics (pseudoneuritis or pseudo-VN). The average age of affected patients in several studies ranged from the mid-50s to early 60 s. This is an age group typically affected with multiple stroke risk factors ${ }^{6} 7$ with high $\mathrm{ABCD} 2$ scores, ${ }^{8 \mathrm{i}}$ and although $\mathrm{VN}$ is presumably the result of an acute viral infection of the superior vestibular nerve $(\mathrm{VN})^{7}$ or an autoimmune disorder, stroke involving the cerebellum or brainstem must be a consideration in the differential diagnosis.

\section{INTRODUCTION}

The AVS is the result of an acute unilateral peripheral or central vestibular lesion that causes a sudden asymmetry of the normal vestibular nuclei neuronal firing rate. Patients with an AVS experience vertigo, nausea and vomiting and head movement intolerance. By and large, the sudden onset of an AVS is associated with severe anxiety, largely due to the continuous and frequently intense illusion of rotation and the associated vasovagal response. ${ }^{9}$ The examiner looks for static (head still) and dynamic (head moving) ocular motor findings, related to alterations of the angular vestibulo-ocular reflex (VOR): nystagmus and head impulse test (HIT) gain ${ }^{10}$; alterations of the vestibulo-spinal reflex: postural unsteadiness and gait imbalance are additional findings. The purpose of this chapter is to discuss the use of 'HINTS' on the diagnosis, triage and management of patients with AVS.

\section{HISTORICAL BACKGROUND}

Generations of neurotologist pioneers in the late 1800 introduced the term nystagmus and searched for clinical features to determine the pathophysiology and localisation of vestibular lesions. Robert Barany in the early 1900 introduced caloric testing as a potential tool to

${ }^{\mathrm{i}}$ A clinical scoring tool used to determine risk of stroke after a transient ischaemic event based on age, hypertension, clinical features, duration and diabetes. The higher the score, the higher the risk. 
study the function of the horizontal semicircular canal ${ }^{11}$; further studies thereafter defined the quantitative parameters of caloric-induced horizontal nystagmus that are widely used to date; however, this diagnostic technique could not be easily performed at bedside. Additional bedside vestibular diagnostic manoeuvres to perform in comatose patients developed. Alfred Bielschowsky in 1939 introduced the 'horizontal and vertical doll's eyes manoeuvre' intended to determine the anatomic and physiological integrity of the brainstem. ${ }^{12}$ Ice water caloric testing used for decades in brain death assessment protocols (2010 AAN Guideline) has no additional practical use at present time. However, the contribution from neuropathological developments, subsequently, highlighted the urgent need for more precise and expeditious diagnostic testing strategies to detect $\mathrm{VN}$ mimics such as cerebellar infarcts associated with malignant oedema and risk of death. ${ }^{13}$ To address these needs, clinicians developed new techniques based on the nystagmus characteristics. Thus, unidirectional horizontal nystagmus that follows Alexander's law and increases with fixation block was the anticipated finding with benign peripheral vestibular lesions. ${ }^{1}$ However, this nystagmus pattern is frequent in central lesions and thus is not a localisation finding. On the other hand, horizontal gaze direction changing nystagmus is a specific sign of a central lesion. In addition, the presence of vertical or purely torsional nystagmus is the result of central lesions as well. ${ }^{14}$ Central nystagmus signs when present have high specificity but unfortunately are infrequent. ${ }^{15}$ Moreover, not infrequently, patients with central lesions have unidirectional nystagmus characteristics identical to those seen with peripheral lesions; therefore there was need to investigate additional signs or a test combination that would yield higher specificity. ${ }^{14}{ }^{16}$ Nystagmus analysis alone provided evidence of central localisation in a small series of initially false negative-stroke MRI patients. ${ }^{17}$

In 1988, Halmagyi and Curthoys described the HIT of the angular VOR. ${ }^{10}{ }^{\text {ii }}$ Loss of the VOR in the AVS is associated with a positive horizontal head impulse test (h-HIT) which has been found consistently as a reliable sign of a peripheral vestibulopathy. ${ }^{18}$ Studies of patients with AVS with high stroke risk factors soon followed and used the horizontal HIT (h-HIT) as a possible sensitive and specific test to provide specific AVS lesion localisation. In an investigator-blinded study, the hypothetical central versus peripheral AVS diagnostic value of h-HIT was tested. Patients with AVS routinely underwent h-HIT testing, prior to imaging or if imaging was performed

\footnotetext{
${ }^{\mathrm{ii}}$ Head movement in the plane of the any of the six semicircular canals (SCC) elicit an excitatory stimulation of ocular motoneurons that generate compensatory eye movements in the direction opposite to the head movement, this reflex enables sustained visual fixation. In vestibulopathy, the angular VOR is impaired as a with decreased or absent excitation of the motoneurons, a visually triggered corrective saccade is necessary to maintain fixation.
}

prior to the h-HIT, the results were concealed to the study investigator. Central AVS lesions were associated with a normal h-HIT, and this was the rule in strokes located in the m-PICA territory, involving either the cerebellum, the medulla or both. ${ }^{14}{ }^{16}$ The clinical value of a normal h-HIT in patients with AVS with a false-negative stroke MRI was compelling enough to warrant repeat imaging which consistently demonstrated the stroke missed in the first MRI. ${ }^{16}$

AICA territory strokes, however, continue to be a diagnostic challenge, as lesions affecting the vestibular root entry, fasciculus or nucleus may potentially be associated with a positive (abnormal h-HIT). To address the potential localisation dilemma, patients with AVS with largeskew deviation (>than 3-prism dioptres), favours central localisation; this finding in my experience is an excellent discriminator. ${ }^{19}$ Skew deviation is a sign of an abnormal otolith-ocular reflex (OOR); thus large amplitude skew deviation and the ocular tilt reaction (OTR) are more common with central lesions. ${ }^{151619}$ While these two signs may be seen rarely with $\mathrm{VN},{ }^{20}$ other peripheral vestibulopathies such as postsurgical cases and severe bacterial inner otitis may be an exception to the rule. ${ }^{21}$

Gufoni integrated both nystagmus direction and skew deviation in a prospective study of patients with predominantly horizontal nystagmus, the fast phase either can be away from the hypertropic eye (called 'downhill') or towards the hypertropic eye (called 'uphill'). Dr Gufoni proposed downhill nystagmus as a central marker and uphill nystagmus as a peripheral marker. ${ }^{22}$ However, when we retrospectively applied this concept to our previously published cohort, the uphill pattern was present in 18 central patients confirmed by imaging. A downhill pattern, on the other hand, was associated with central lesions. ${ }^{16} 19$

The triad of h-HIT, horizontal, direction changing nystagmus and skew deviation compared with the results of MRI performed within 48 hours after symptom onset revealed that the combined value of the three ocular motor tests; provided greater sensitivity (100\%) and specificity $(96 \%) \cdot{ }^{16}$ In the first submission, the title of the manuscript was 'Subtle Eye Signs in AVS differentiate central from peripheral vestibular lesions,' however, one manuscript reviewer suggested a change in the title, and David Newman-Toker, M.D., PhD then coined the acronym: 'HINTS', widely used since then. The acronym refers to HI: Head Impulse, N: Nystagmus direction and TS: Testing Skew. In addition, a second acronym and mnemonic: INFARCT summarises the application of HINTS: IN: Impulse normal, FA: Fast Alternates (referring to the nystagmus fast phase) and RCT: Refixation on cover test (skew deviation). ${ }^{16}$

Since the introduction of HINTS, there have been several papers using the diagnostic triad (table 1). HINTS has been more sensitive that DWI MRI in several reports, particularly in lacunar strokes. ${ }^{23}{ }^{24}$ I intend to discuss the results of these studies. Will review in addition, the possibility of additional signs pointing to a central localisation 
Table 1 HIT positive: in favour of stroke diagnosis with either normal gain, vertical or purely torsional nystagmus or with horizontal, direction changing nystagmus with horizontal gaze positions

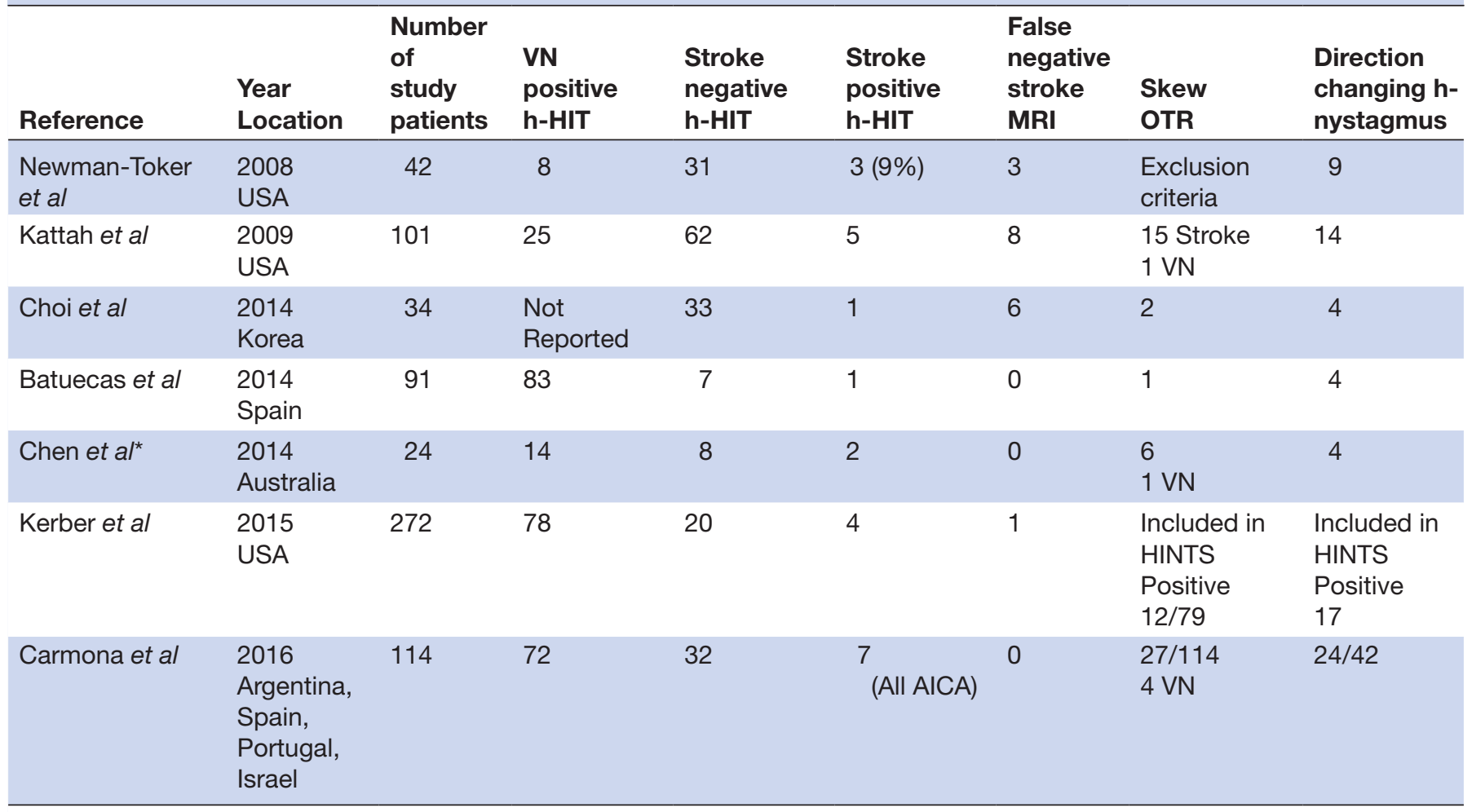

HIT, head impulse test; OTR, ocular tilt reaction; VN, vestibular neuritis.

under the family of HINTS PLUS signs. ${ }^{15} 19$ Importantly, graphic recording of h-HIT has been possible for the last few years, using Video head impulse goggles (vHIT). There are several advantages of these recordings which provide VOR gain values for all six semicircular canals, vHIT records both overt and covert saccades, ${ }^{\text {iii }}$ in addition, it records the number of corrective saccades, their latency and the detection of artefacts that may lead to equivocal clinical responses. ${ }^{25}$ We will discuss these new techniques and their potential application. The pilot phase of a clinical trial (AVERT Acute Video-Oculography for Vertigo in Emergency Rooms for Rapid Triage) to analyse the diagnostic and triaging impact of a vHIT protocol guided management of patient with AVS began in January 2016. David Newman Toker, M.D., PhD is the PI in the study with a three-site recruiting locations.

We will proceed with the description of each of the HINTS triad components as it is routinely performed in the evaluation of patients with AVS.

- Nystagmus direction analysis: Horizontal nystagmus in peripheral and central lesions. Pseudo-spontaneous nystagmus. Gaze induced, direction Changing Nystagmus, Head Shaking nystagmus, pure torsional

\footnotetext{
iii These visual triggered saccades occur when the angular VOR is decreased as a result of primary vestibular dysfunction (periphery, root entry or vestibular nucleus). Overt Saccade: Catch-up saccade noted after a head impulse test. Covert saccade: Corrective saccade occurs during the head impulse test and cannot be detected without vHIT recording.
}

and vertical nystagmus in patients with AVS with stroke risk factors.

Primary position spontaneous horizontal nystagmus that increases with fixation removal and follows Alexander's lawiv is the rubric of $\mathrm{VN}$ and other peripheral vestibulopathies. The clinical examination of patients with AVS should begin therefore by analysing the direction of the nystagmus in all cardinal positions of gaze, followed by testing the effect of fixation block in each gaze position. Direct ophthalmoscopy and monocular occlusion introduced by David Zee, M.D. is very effective, ${ }^{26}$ the penlight test, described by Newman-Toker et al is also a helpful bedside technique to test the effect of fixation block. The patient covers one eye while the examiner shines a penlight in the fellow eye. The retinal bleach effect from sustained light exposure blocks fixation of the uncovered eye and enhances a latent vestibular nystagmus. ${ }^{27}$ Frenzel's goggles and other recently designed portable goggles are additional options. ${ }^{28}$ Typically, peripheral vestibular lesions are associated with unidirectional nystagmus that increases in the gaze direction of the fast phase (Alexander's law). This nystagmus pattern, however, may be seen with cerebellar or brainstem lesions. ${ }^{141619}$ Clinicians must be alert to detect 'pseudo-neuritis' presentations due to stroke and other acute neurological syndromes. In the majority of the studies using the h-HIT, VN is associated

${ }^{\text {iv }}$ The amplitude of the nystagmus increases in the gaze-direction of the primary position nystagmus fast phase. 
with a positive HIT, unidirectional horizontal nystagmus that follows Alexander's law, a negative MRI and a typical benign clinical course.

Of note, the possibility of 'pseudo-spontaneous nystagmus in the primary position of gaze', due to horizontal canal paroxysmal positional vertigo becomes evident with a horizontal nystagmus fast phase direction change prompted by a forward head pitch. ${ }^{29}$ This nystagmus direction switch is typical for horizontal canal (hc) benign paroxysmal positional vertigo (BPPV).

To confirm hc BPPV, I observe the nystagmus elicited by head roll in the supine position. Two horizontal nystagmus patterns may occur: the first type and most common, is a rather robust, fast velocity nystagmus in the direction of the head roll (geotropic); the second type involves a fast nystagmus phase beating away from the direction of the head roll (apogeotropic). Both types should be treated with canal repositioning manoeuvres and do not require additional testing. An apogeotropic nystagmus type may be seen with central lesions ${ }^{30}$ and less commonly with geotropic variants. ${ }^{31}$

As mentioned before, Hotson and Baloh identified horizontal nystagmus with fast phase direction change as a specific central sign. ${ }^{1}$ This finding, coupled with a normal h-HIT, is compelling enough to repeat imaging when a clinical diagnosis of stroke is conflicted by an initially, false negative MRI study. Peripheral lesions do not cause this nystagmus pattern. ${ }^{14} 16$ To explain the gaze induced direction change, ${ }^{\mathrm{vi}}$ impairment of gaze-holding pathways (neural integrator) in the cerebellum, the brainstem or their connecting fibres in the cerebellar peduncles must be compromised. ${ }^{115}$ Clinicians may rely with confidence on horizontal, direction changing nystagmus as a central localisation sign.

Rebound horizontal nystagmus ${ }^{\mathrm{vii}}$ was not common in the ischaemic lesions series summarised in table $1,{ }^{14} 16$ it may be seen with cerebellar or extra-axial brainstem lesions and is always a central sign. ${ }^{32}$ Clinicians should also be aware of the fact that demyelination, infectious and nutritional/toxic aetiologies may be responsible for a central AVS. ${ }^{33}{ }^{34}$ Imaging, clinical examination and laboratory testing will identify most of these possibilities. Head shaking for a few seconds usually increases the amplitude of the primary nystagmus; however, variations such as posthead shaking horizontal nystagmus direction change or its conversion to vertical nystagmus (perverted nystagmus) are central signs. This nystagmus elicitation

\footnotetext{
${ }^{\mathrm{v}}$ The head is rapidly turned $90^{\circ}$ to either side while supine, this manoeuver excites the horizontal semicircular and inhibits the contralateral canal.

${ }^{\mathrm{vi}}$ Nystagmus elicited by changing the direction of gaze: horizontal or vertical. Of note the nystagmus fast phase does not have to match the Gaze direction, for example a right-beat nystagmus may be present in upgaze.

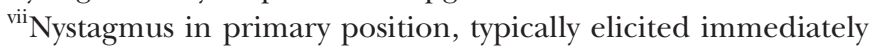
after sustained lateral gaze. It beats in direction opposite to the GEN and lasts usually 20-30 s.
}

technique was the only central localisation in one patient from a previous AVS, AICA stroke series. ${ }^{35}$ Positional testing (Dix Hallpike or lateral head roll in the supine position) does not change the h-primary gaze nystagmus direction. Furthermore, any direction change will also point to central localisation. ${ }^{36}$ Finally, primary gaze vertical or purely torsional nystagmus point to central localisation ${ }^{15}$ and was relatively uncommon in most AVS series summarised in table 1.

\section{The h-HIT test in patients with AVS}

Clinicians use the h-HIT extensively since its inception in both unilateral and bilateral vestibulopathy. ${ }^{10} 18$ The normal response to a rapid, passive eye movement as a subject fixates on a central target is an equal and opposite eye movement of the same magnitude (Gain: ratio of head velocity/eye velocity is 1.0 ). The head thrusts may be performed with the head moving towards or away from centre position, the VOR gain does not change. ${ }^{37}$ In the event of a peripheral vestibular lesion involving the primary VOR pathway, the acceleration signal to move the eyes is impaired, resulting in gain loss (less than 1.0), consequently the eyes cannot stay on target and once the head stops a refixation saccade is thus required. Typically the vHIT performed in acute peripheral AVS shows a significant right versus left asymmetry and a conspicuous unidirectional corrective saccade. ${ }^{38}$

Choi et al from Korea reported in 2014 their experience with HINTS in 34 patients with isolated vestibular syndromes due to lacunar stroke (table 1). They found central ocular motor signs in 33 patients and had 6 patients with initially false negative DWI MRI studies. ${ }^{23}$ Batuecas et al from Spain in 2014 also used the HINTS diagnostic triad in 91 patients with AVS, 8 of them had a stroke and 7 had central HINTS. No false negative MRI studies were reported. ${ }^{39}$ The majority of the remaining patients with peripheral lesions had peripheral HINTS results; a small number of peripheral lesions had a normal h-HIT, suggesting peripheral vestibular diagnoses other than VN. Chen et alfrom Australia in 2011 and later in 2014 studied patients with AVS, recording the h-HIT using magnetic search coils, which is the gold standard for ocular motor recording accuracy. All their patients with stroke had positive MRIs. Their contribution outlined the value added by v-HIT recordings to the analysis of the h-HIT. Significant gain asymmetry occurs in peripheral lesions (in particular VN). In addition, such asymmetry occurs in AICA strokes with a peripheral vestibular h- HIT pattern, although of lesser magnitude. Other patterns occur in AICA strokes with a central pattern and PICA strokes. Lateral medullary lesions were not part of the analysis. ${ }^{40}{ }^{41}$ In 2015 Kerber et $a t^{42}$ reported their experience with HINTS in a large cohort from the University of Michigan in Ann Arbor. They found a central HINTS pattern in 20 out of 29 patients who had stroke. Of note, seven of their patients did not have spontaneous nystagmus, which may be a special diagnostic category in which the HINTS triad needs additional investigation. 

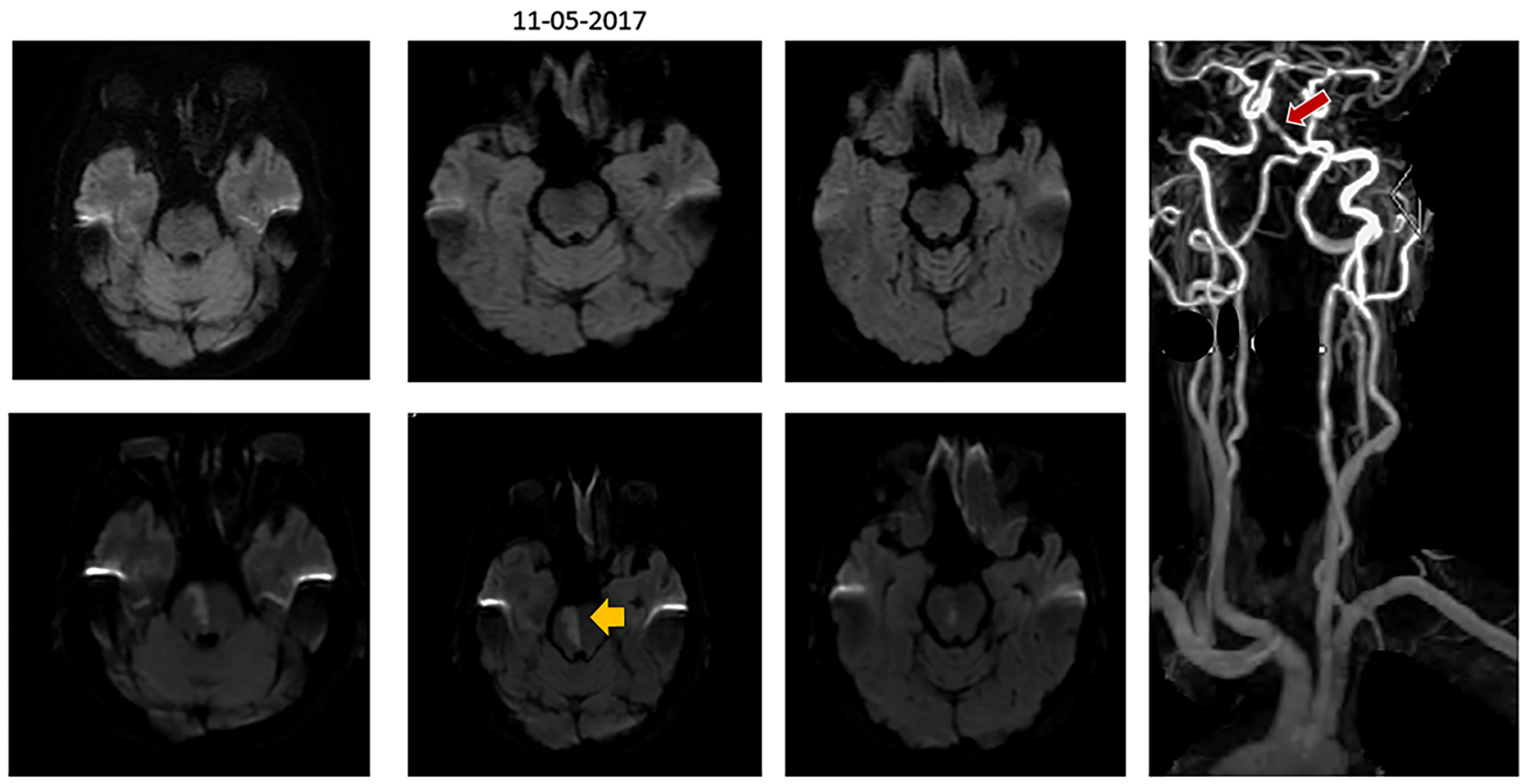

11-06-2017

Figure 1 Axial DWI MRI. The top three panels represent serial images obtained when the patient was asymptomatic. The lower three panels obtained while symptomatic, about 10 hours later with primary gaze upbeat nystagmus and a right internuclear ophthalmoplegia. The red arrow points to the mid-basilar stenosis and occlusion of the lumen of a paramedian penetrator artery. The yellow arrow points to the midline pontine tegmentum/basis pontis stroke.

However, their 22 patients with had a concordant central HINTS results. In addition, the infarction detected by MRI was possibly an incidental finding, not responsible for the ocular motor signs (supratentorial localisation, etc). In their series, they had only one false negative stroke MRI. Potential limitations of this study include mixed-skill rater examiners and only fair interrater agreement (Kappa: 0.24-0.40). ${ }^{42}$ Moreover, the classification of non-stroke central aetiologies (with central HINTS results) mixed with peripheral vestibulopathies might be confusing from the localisation viewpoint. ${ }^{43}$ This being said, the large size of the Michigan's AVS cohort, active and passive surveillance methods to capture patients with acute dizziness, MRI studies in 195 patients (either clinical or research) and correct stroke prediction in 20/22 patients with AVS with nystagmus highlights the diagnostic usefulness of the triad. Ischaemic lesions in the cerebellar flocculus may cause an impaired HIT $^{44}{ }^{45}$; these patients had a robust contralesional OTR and impaired horizontal pursuit, both suggested a central HINTS category. In selective peripheral vestibular syndromes due to infection or surgical inner ear procedures, an OTR may be present ${ }^{2146}$; however, in my personal experience with $\sim 100$ patients with VN, I never encountered an OTR (unpublished observation).

As a general comment, AICA territory strokes may have a normal h-HIT if the ischaemic lesions spares the integrity of the angular $\mathrm{h}-\mathrm{VOR}^{47}$ (figure 1). Simultaneous AICA/PICA strokes may have also a unilaterally abnormal h-HIT due to involvement of the medial vestibular nucleus. $^{36}{ }^{48-52}$ Ischaemic lesion affecting the nucleus prepositus hypoglossi have a contralesional decreased VOR gain and an asymmetric horizontal, direction changing nystagmus. ${ }^{5152}$

From the aforementioned, it is clear that the use of the manual h-HIT clinically and the utilisation of the v-HIT technology in the ED and at bedside in the ward or intensive care unit will affect diagnosis of AVS and select patients requiring imaging studies. The HINTS triad is particularly helpful when evaluating patients with nystagmus. The h-HIT/nystagmus analysis is a combined index of semicircular canal function for combined analysis. An abnormal h-HIT (with decreased gain and a corrective refixation saccade) in association with unidirectional h-nystagmus will be the signature sign of peripheral vestibulopathies. The forthcoming results of multiple trials in progress will predictably add further information to the epidemiology of AVS and the impact of HINTS applied to dizzy patients evaluated in the ED.

\section{Skew deviation}

Skew deviation refers to a vertical ocular misalignment in the primary position of gaze reflecting an impaired OOR. Patients with skew deviation larger exceeding prism-dioptres generally report vertical binocular diplopia, unless their vertical fusion power is greater than normal. In most patients with AVS, however, the overwhelming effect of large amplitude nystagmus, postural unsteadiness and vasovagal symptoms take precedence over other symptoms, therefore, diplopia is not generally a chief complaint in the early 
AVS. It is therefore, important to add the cross-cover test routinely in the AVS examination protocol even in patients without diplopia.

Ocular motor examination of the OOR begins by asking the patient to fixate on a central target and observing the vertical position of the eyes. Occasionally, large amplitude skew is obvious by simple observation. More commonly, however, skew manifests when the examiner tests fixation with the alternate cross-cover. Whereas patients with normal ocular alignment do not have a refixation movement, patients with an impaired OOR have disconjugate vertical refixating saccades to stay on target (skew). An impaired OOR results in conjugate ocular torsion but disconjugate vertical alignment. Accordingly, one eye is lower: hypotropic and the fellow eye is higher: hypertropic. This is the most common OOR abnormality found in patients with ischaemic stroke. ${ }^{53}$ This technique identifies skew even in patients with intense nystagmus. In addition, analysis of the head position at rest should be observed; as sustained head tilt may be seen in association with skew. ${ }^{54}$ The cross-cover technique described in our study measured the degree of skew deviation (average amplitude of 9.9 prism-dioptres, with a range from 3 to 20 prism-dioptres).${ }^{16}$ Finally, digital fundus photography if available may provide evidence of conjugate ocular torsion, the third OTR component. VN affects more frequently the superior vestibular nerve, which provides most of the innervation to the utricle; thus, low amplitude skew deviation is infrequent in $\mathrm{VN}$, and when present is generally of low amplitude. Keane in 1975 defined the lateralisation value of skew deviation. Whereas the hypotropic eye is ipsilesional in peripheral vestibular and medullary lesions, it is generally contralesional in pontine and midbrain lesions. ${ }^{535}$

The vHIT measures all HINTS triad components. The recording performed at bedside is very sensitive in detecting and quantitating skew deviation. HINTS tracings will provide an objective measure of canal and otolith function and set a baseline for further follow-up. It may also be useful in understanding the process of vestibular compensation in peripheral and central vestibular lesions.

\section{HINTS PLUS}

In 2013, HINTS PLUS hearing loss due to cochlear or brainstem ischaemia was introduced as a new sign that could potentially increase diagnostic accuracy. ${ }^{15}$ The vascular supply to the inner ear, the fourth-nerve complex (cochlear, superior and inferior vestibular and facial nerves) in the cerebellopontine angle, the cochlear nerve root entry and the cochlear nucleus derives frequently from AICA ( 80\% of cases), the basilar artery ( 15\%$20 \%$ ) and only rarely via PICA. Hearing loss/deafness in patients with AVS is infrequent in acute stroke. ${ }^{56}$ A thorough neurological evaluation should follow the HINTS examination. Severe truncal ataxia adds sensitivity to HINTS PLUS. ${ }^{57}$ In general, entertain the possibility of a central lesion in patients who are acutely unable to stand and walk independently.

\section{Transient ischaemic attacks: TIA}

The evaluation of the patient with 'continuous vertigo' in general leads to a precise diagnosis. The challenge is the evaluation of episodic vascular vertigo. Dizziness was the most common symptom of posterior circulation compromise in the New England Posterior Circulation Registry. ${ }^{58}{ }^{59}$ A recent case highlights this challenge. A 61-year-old woman with an ABCD2 score of '4' presented with a 24 hours history of three, recurrent vertigo episodes that lasted $\sim 30 \mathrm{~min}$ each, associated with imbalance, nausea and vomiting (figure 1). Her examination was normal and the initial MRI, including DWI was unremarkable, she did not have an MRA; she was able to walk out of the hospital without assistance. On awakening the next morning, she had severe ataxia, oscillopsia and diplopia. On examination, she had primary gaze upbeat nystagmus and a right internuclear ophthalmoplegia. MRI showed a pontine stroke, due to occlusion of the lumen of a penetrator branch of the basilar artery. The lesson learnt is the need to include vascular imaging in these high-risk patients, either CT angiography or MRA.

\section{CONCLUSION}

The potential diagnostic impact of vHIT by objectively analysing HINTS $^{52} 60$ which has been described as an ECG of the eyes ${ }^{60}$ will enable in future years development of updated management guidelines which combine improved diagnostic accuracy and patient safety with effective, low-cost management.

Funding The authors have not declared a specific grant for this research from any funding agency in the public, commercial or not-for-profit sectors.

Competing interests GN Otometrics Co. Trastup, Denmark loaned us research equipment in 2012.

Patient consent Not required.

Provenance and peer review Commissioned by the CSA; externally peer reviewed.

Data sharing statement There are no additional data available.

Open access This is an open access article distributed in accordance with the Creative Commons Attribution Non Commercial (CC BY-NC 4.0) license, which permits others to distribute, remix, adapt, build upon this work non-commercially, and license their derivative works on different terms, provided the original work is properly cited and the use is non-commercial. See: http://creativecommons.org/ licenses/by-nc/4.0/

(c) Article author(s) (or their employer(s) unless otherwise stated in the text of the article) 2018. All rights reserved. No commercial use is permitted unless otherwise expressly granted.

\section{REFERENCES}

1. Hotson JR, Baloh RW. Acute vestibular syndrome. N Engl J Med 1998;339:680-5.

2. Tarnutzer AA, Berkowitz AL, Robinson KA, et al. Does my dizzy patient have a stroke? A systematic review of bedside diagnosis in acute vestibular syndrome. CMAJ 2011;183:E571-92.

3. Newman-Toker DE. Missed stroke in acute vertigo and dizziness: It is time for action, not debate. Ann Neurol 2016;79.

4. Caplan LR, Wityk RJ, Glass TA, et al. New England medical center posterior circulation registry. Ann Neurol 2004;56:389-98. 
5. Searls DE, Pazdera L, Korbel E, et al. Symptoms and signs of posterior circulation ischemia in the new England medical center posterior circulation registry. Arch Neurol 2012;69:346-51.

6. Mandalà M, Nuti D. Long-term follow-up of vestibular neuritis. Ann N Y Acad Sci 2009;1164:427-9.

7. Strupp M, Zingler VC, Arbusow V, et al. Methylprednisolone, valacyclovir, or the combination for vestibular neuritis. N Engl J Med 2004;351:354-61.

8. Navi BB, Kamel H, Shah MP, et al. Application of the ABCD2 score to identify cerebrovascular causes of dizziness in the emergency department. Stroke 2012;43:1484-9.

9. Baloh RW HM. Disorders of the vestibular system. UK: Oxford University Press, 1996:175-7.

10. Halmagyi GM, Curthoys IS. A clinical sign of canal paresis. Arch Neurol 1988;45:737-9.

11. Lopez C, Blanke O. Nobel Prize centenary: Robert Bárány and the vestibular system. Curr Biol 2014;24:R1026-8.

12. Lippmann O. Alfred Bielschowsky's 1940 legacy for neuroophthalmology. J Neuroophthalmol 1998;18:227-30.

13. Duncan GW, Parker SW, Fisher CM. Acute cerebellar infarction in the PICA territory. Arch Neurol 1975;32:364-8.

14. Newman-Toker DE, Kattah JC, Alvernia JE, et al. Normal head impulse test differentiates acute cerebellar strokes from vestibular neuritis. Neurology 2008;70(24 Pt 2):2378-85.

15. Newman-Toker DE, Curthoys IS, Halmagyi GM. Diagnosing stroke in acute vertigo: the HINTS family of eye movement tests and the future of the "Eye ECG". Semin Neurol 2015;35:506-21.

16. Kattah JC, Talkad AV, Wang DZ, et al. HINTS to diagnose stroke in the acute vestibular syndrome: three-step bedside oculomotor examination more sensitive than early MRI diffusion-weighted imaging. Stroke 2009;40:3504-10.

17. Oppenheim C, Stanescu R, Dormont D, et al. False-negative diffusion-weighted MR findings in acute ischemic stroke. AJNR Am J Neuroradiol 2000;21:1434-40.

18. Schmid-Priscoveanu A, Böhmer A, Obzina $\mathrm{H}$, et al. Caloric and search-coil head-impulse testing in patients after vestibular neuritis. $J$ Assoc Res Otolaryngol 2001;2:72-8.

19. Newman-Toker DE, Kerber KA, Hsieh YH, et al. HINTS outperforms $A B C D 2$ to screen for stroke in acute continuous vertigo and dizziness. Acad Emerg Med 2013;20:986-96.

20. Safran $A B$, Vibert $D$, Issoua $D$, et al. Skew deviation after vestibular neuritis. Am J Ophthalmol 1994:118:238-45.

21. Kung NH, Van Stavern GP, Gold DR. HINTS in the acute vestibular syndrome: pearls and pitfalls. J Neuroophthalmol 2018;38:244-250.

22. Gufoni M. Uphill/downhill nystagmus. Acta Otorhinolaryngol Ital 2017;37:513-8.

23. Choi JH KH, Dong K, Kim MJ, et al. Isolated vestibular syndrome in posterior circulation stroke. Neurology Clinical Practice 2014;4:410-8.

24. Saber Tehrani AS, Kattah JC, Mantokoudis G, et al. Small strokes causing severe vertigo: frequency of false-negative MRIs and nonlacunar mechanisms. Neurology 2014;83:169-73.

25. Mantokoudis G, Tehrani AS, Wozniak A, et al. VOR gain by head impulse video-oculography differentiates acute vestibular neuritis from stroke. Otol Neurotol 2015;36:457-65.

26. Zee DS. Ophthalmoscopy in examination of patients with vestibular disorders. Ann Neurol 1978;3:373-4.

27. Newman-Toker DE, Sharma P, Chowdhury M, et al. Penlight-cover test: a new bedside method to unmask nystagmus. J Neurol Neurosurg Psychiatry 2009;80:900-3.

28. Strupp M, Fischer $C$, Han $\beta$ L, et al. The takeaway Frenzel goggles: a Fresnel-based device. Neurology 2014;83:1241-5.

29. Asprella-Libonati G. Pseudo-spontaneous nystagmus: a new sign to diagnose the affected side in lateral semicircular canal benign paroxysmal positional vertigo. Acta Otorhinolaryngol Ital 2008;28:73-8.

30. Choi JY, Glasauer S, Kim JH, et al. Characteristics and mechanism of apogeotropic central positional nystagmus. Brain 2018:762-75.

31. Yang TH, Oh SY. Geotropic central paroxysmal positional nystagmus in a patient with human immunodeficiency virus encephalopathy. $J$ Neuroophthalmol 2014;34:159-61.

32. Zee DS, Yamazaki A, Butler PH, et al. Effects of ablation of flocculus and paraflocculus of eye movements in primate. $J$ Neurophysiol 1981;46:878-99.

33. Pula JH, Newman-Toker DE, Kattah JC. Multiple sclerosis as a cause of the acute vestibular syndrome. $J$ Neurol 2013;260:1649-54.
34. Kattah JC DS, Pula JH, Mantokoudis G, et al. Vestibular signs in non-encephalopathic Wernicke's disease. Neurol Clin Practice 2013;3:460-7.

35. Huh YE, Koo JW, Lee H, et al. Head-shaking aids in the diagnosis of acute audiovestibular loss due to anterior inferior cerebellar artery infarction. Audiol Neurootol 2013;18:114-24.

36. Shaikh AG, Miller BR, Sundararajan S, et al. Gravity-dependent nystagmus and inner-ear dysfunction suggest anterior and posterior inferior cerebellar artery infarct. J Stroke Cerebrovasc Dis 2014;23:788-90.

37. Schubert MC, Mantokoudis G, Xie L, et al. Acute VOR gain differences for outward vs. inward head impulses. J Vestib Res 2014:24(5-6):397-402.

38. Weber KP, MacDougall HG, Halmagyi GM, et al. Impulsive testing of semicircular-canal function using video-oculography. Ann N Y Acad Sci 2009;1164:486-91

39. Batuecas CA, Y.G, Sanchez BC, et al. Application of the HINTS protocol. Rev Neurol 2014;59:349-53.

40. Chen L, Todd M, Halmagyi GM, et al. Head impulse gain and saccade analysis in pontine-cerebellar stroke and vestibular neuritis. Neurology 2014;83:1513-22.

41. Chen L, Lee W, Chambers BR, et al. Diagnostic accuracy of acute vestibular syndrome at the bedside in a stroke unit. $J$ Neurol 2011;258:855-61.

42. Kerber KA, Meurer WJ, Brown DL, et al. Stroke risk stratification in acute dizziness presentations: A prospective imaging-based study. Neurology 2015;85:1869-78.

43. Choi SY, Kim HJ, Kim JS. Chasing dizzy chimera: diagnosis of combined peripheral and central vestibulopathy. J Neurol Sci 2016;371:69-78

44. Park HK, Kim JS, Strupp M, et al. Isolated floccular infarction: impaired vestibular responses to horizontal head impulse. J Neurol 2013;260:1576-82.

45. Yacovino DA, Akly MP, Luis L, et al. The floccular syndrome: dynamic changes in eye movements and vestibulo-ocular reflex in isolated infarction of the cerebellar flocculus. Cerebellum 2018;17:122-131.

46. Halmagyi GM, Gresty MA, Gibson WP. Ocular tilt reaction with peripheral vestibular lesion. Ann Neurol 1979;6:80-3.

47. Kattah JC, Nair D, Talkad A, et al. A case of vestibular and oculomotor pathology from bilateral AICA watershed infarcts treated with basilar artery stenting. Clin Neurol Neurosurg 2013:115:1098-101.

48. Kim JS, Choi KD, Oh SY, et al. Medial medullary infarction: abnormal ocular motor findings. Neurology 2005;65:1294-8.

49. Lee H, Kim JS, Chung EJ, et al. Infarction in the territory of anterior inferior cerebellar artery: spectrum of audiovestibular loss. Stroke 2009;40:3745-51.

50. Kim HA, Lee H. Isolated vestibular nucleus infarction mimicking acute peripheral vestibulopathy. Stroke 2010;41:1558-60.

51. Kim SH, Zee DS, du Lac S, et al. Nucleus prepositus hypoglossi lesions produce a unique ocular motor syndrome. Neurology 2016;87:2026-33.

52. Choi JY, Kim HJ, Kim JS. Recent advances in head impulse test findings in central vestibular disorders. Neurology 2018;90:602-12.

53. Keane JR. Ocular skew deviation. Analysis of 100 cases. Arch Neurol 1975;32:185-90.

54. Kattah JC, Dagi TF. Compensatory head tilt in upbeating nystagmus. J Clin Neuroophthalmol 1990;10:27-31.

55. Brodsky MC, Donahue SP, Vaphiades M, et al. Skew deviation revisited. Surv Ophthalmol 2006;51:105-28.

56. Huang $\mathrm{MH}$, Huang $\mathrm{CC}$, Ryu SJ, et al. Sudden bilateral hearing impairment in vertebrobasilar occlusive disease. Stroke 1993;24:132-7.

57. Carmona S, Martínez C, Zalazar G, et al. The Diagnostic accuracy of truncal ataxia and HINTS as cardinal signs for acute vestibular syndrome. Front Neurol 2016;7:125.

58. Caplan L, Chung CS, Wityk R, et al. New England medical center posterior circulation stroke registry: I. Methods, data base, distribution of brain lesions, stroke mechanisms, and outcomes. $J$ Clin Neurol 2005;1:14-30.

59. Caplan LR. Vertebrobasilar ischemia and hemorrhage: clinical fidnings. Diagnosis and management of posterior circulation disease. UK: Cambridge University Press, 2015.

60. Newman-Toker DE, Saber Tehrani AS, Mantokoudis G, et al. Quantitative video-oculography to help diagnose stroke in acute vertigo and dizziness: toward an ECG for the eyes. Stroke 2013;44:1158-61. 